\title{
Red Giant Oscillations: Stellar Models and Mode Frequency Calcula- tions
}

\author{
A. Jendreieck ${ }^{1, \star}$, A. Weiss ${ }^{1}$, V. Silva Aguirre ${ }^{2}$, J. Christensen-Dalsgaard ${ }^{2}$, R. Handberg ${ }^{2}$, G. Ruchti ${ }^{1}$, \\ C. Jiang ${ }^{2}$, and A. Thygesen ${ }^{3}$ \\ 1 Max Planck Institute for Astrophysics, Karl-Schwarzschild-Str. 1, 85748, Garching bei München, Germany \\ 2 Stellar Astrophysics Centre, Department of Physics and Astronomy, Aarhus University, Ny Munkegade 120, \\ DK-8000 Aarhus C, Denmark \\ 3 Zentrum für Astronomie der Universität Heidelberg, Landessternwarte, Heidelberg, Germany
}

Received 22 August 2012, accepted 18 October 2012

Published online?

Key words stars: oscillations; stars: evolution

We present preliminary results on modelling KIC 7693833, the so far most metal-poor red-giant star observed by Kepler. From time series spanning several months, global oscillation parameters and individual frequencies were obtained and compared to theoretical calculations. Evolution models are calculated taking into account spectroscopic and asteroseismic constraints. The oscillation frequencies of the models were computed and compared to the Kepler data. In the range of mass computed, there is no preferred model, giving an uncertainty of about $30 \mathrm{~K}$ in $T_{\text {eff }}, 0.02 \operatorname{dex}$ in $\log g, 0.7 R_{\odot}$ in radius and of about $2.5 \mathrm{Gyr}$ in age.

(c) WILEY-VCH Verlag GmbH \& Co. KGaA, Weinheim

\section{Introduction}

Red giants have a broad spectrum of acoustic oscillation modes which are excited by an extended convective envelope (e.g. Montalbán et al. 2010; Mosser et al. 2011). Also, as the density in the helium core is quite large, g-modes with frequencies close to those of the acoustic modes can interact with the latter, bringing on the presence of so-called mixed modes (Di Mauro et al. 2011). Those modes with a more p-mode character, propagating with smaller inertia, can reach the surface where they are observed. As they have g-mode character in the centre, they provide important information about the deep interior of red giants. The high sensitivity of the CoRoT and Kepler space missions made it possible to observe non-radial oscillations in a large sample of red giants (De Ridder et al., 2009) and the observation of such mixed modes (e.g. Di Mauro et al. 2011). The study of evolved stars is important as it leads to better constraints on stellar evolution models, since the uncertainties in stellar structure properties accumulate with age (De Ridder et al. 2009). Processes not well understood like convective overshooting, rotational mixing and diffusion during the hydrogen-burning phase determines the mass of the helium core in the giant phase and also the age of the star (Aerts et al. 2008).

\footnotetext{
* Corresponding author: e-mail: ajendreieck@mpa-garching.mpg.de
}

Table 1 Basic parameters of KIC 7693833 determined and used in the present analysis.

\begin{tabular}{cc}
\hline \hline \multicolumn{2}{c}{ Basic Parameters of KIC 7693833 } \\
\hline$T_{\text {eff }}$ & $5119 \pm 140 \mathrm{~K}$ \\
$\log \mathrm{g}$ & $2.4 \pm 0.2 \mathrm{dex}$ \\
{$[\mathrm{Fe} / \mathrm{H}]$} & $-2.20 \pm 0.1 \mathrm{dex}$ \\
$\alpha$-enhancement & $0.2 \mathrm{dex}$ \\
$\nu_{\max }$ & $32.2 \pm 2.0 \mu \mathrm{Hz}$ \\
$\Delta \nu$ & $4.06 \pm 0.2 \mu \mathrm{Hz}$ \\
\hline \hline
\end{tabular}

\section{Observations}

We have analyzed the red giant KIC 7693833 observed by Kepler during the Q1-Q10. The frequency spectrum spans a range from $20 \mu \mathrm{Hz}$ to $50 \mu \mathrm{Hz}$ with 19 modes identified as $l=0,1,2$ with a $\nu_{\max }=32.2 \mu \mathrm{Hz}$ and $\Delta \nu=4.06 \mu \mathrm{Hz}$. The target KIC 7693833 is a metal-poor star classified $([\mathrm{Fe} / \mathrm{H}]=-2.2 \mathrm{dex})$ as ascending the red-giant branch according to the classification given by Bedding et al. (2011). Spectroscopic analysis was performed by Thygesen et al. (2012) and a $T_{\text {eff }}$ of about $4800 \mathrm{~K}$ was determined. We reanalyzed the spectra considering NLTE effects and found a $T_{\text {eff }}$ of about $5100 \mathrm{~K}$. We also found indications of a constant $\alpha$-enhancement of 0.2 dex. From the corrected Kepler lightcurves we extracted the global seismic parameters $\Delta \nu$ and $\nu_{\max }$, as well as individual frequencies for modes of degree $l=0,1,2$. The basic stellar parameters are summarized in Table1. 


\section{Models}

Making use of the observed constraints on the asteroseismic parameters and the effective temperature, we first estimated a preliminary mass using the asteroseismic scaling relation (Brown et al. 1991; Kjeldsen \& Bedding 1995):

$$
\frac{M}{M_{\odot}}=\left(\frac{\Delta \nu}{\Delta \nu_{\odot}}\right)^{-4}\left(\frac{\nu_{\max }}{\nu_{\max , \odot}}\right)^{3}\left(\frac{T_{\mathrm{eff}}}{T_{\mathrm{eff}, \odot}}\right)^{3 / 2}
$$

resulting in a mass of $1.17 M_{\odot}$. With this information, a grid of models was calculated for masses from $1.00-1.20$ $M_{\odot}$ in steps of $0.01 M_{\odot}$ with the GARSTEC evolutionary code (Weiss \& Schlattl 2008).

All the models were computed with the same input parameters. OPAL 2005 (Rogers \& Nayfonov 2002) equation of state were used together with NACRE (Angulo et al. 1999) reaction rates. The metallicity was calculated considering the $\alpha$-enhancement according to Salaris et al. (1997), giving a $Z=1.6 \times 10^{-4}$. We used the He primordial content $Y=0.248$ derived by Steigman (2010). Convection is treated according to the mixing-length theory (MLT) (Böhm-Vitense 1958) and with the parameter $\alpha_{\mathrm{MLT}}=$ 1.744 calibrated for the Sun.

The oscillation frequencies were calculated with the ADIPLS package (Christensen-Dalsgaard 2008) for modes of angular degree $l=0,1$ and 2 for all models that satisfied $\Delta \nu$ observed according to the scaling relation:

$$
\frac{\Delta \nu}{\Delta \nu_{\odot}}=\left(\frac{M}{M_{\odot}}\right)^{1 / 2}\left(\frac{L}{L_{\odot}}\right)^{-0.75}\left(\frac{T_{\text {eff }}}{T_{\text {eff }, \odot}}\right)^{3} .
$$

Afterwards, a thinner time step was set to find the models that matched the lowest $l=0$ mode observed within the error bars.

\section{Results to Date}

We compared the calculated and observed frequencies. As the calculated frequency spectrum is heavily populated, only the frequencies with minimum mode inertia were chosen to be compared because these modes have the highest amplitudes at the surface and hence are the most probable to be observed (e.g. Christensen-Dalsgaard et al. 1995; Dupret et al. 2009). Figure 1 shows mode inertia plotted versus frequency for a model with $1 M_{\odot}$. Radial modes are represented as diamonds, $l=1$ modes as triangles and $l=2$ modes as squares.

Figure 2 shows the difference between calculated and observed frequencies, for some of the models with masses 1.0 and $1.2 \mathrm{M}_{\odot}$. The error bars are representing the errors from the observational data. The frequency differences are smaller than $1.0 \mu \mathrm{Hz}$ for all models considered up to now, having no preferred model. Figure 3 shows the échelle diagrams for the same models. Open symbols are the frequencies computed from models and filled circles are representing the observational data. Both models show good agreement with data, showing again no preferred model.

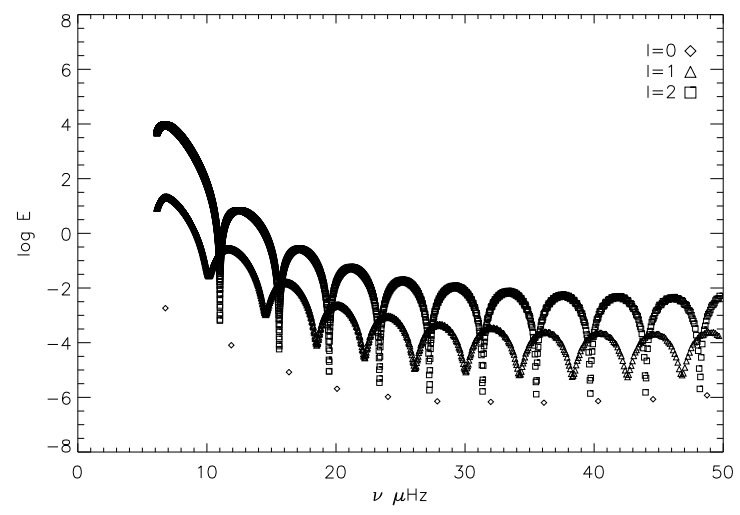

Fig. 1 Mode inertia as function of frequency for modes $l=0$ (diamonds), $l=1$ (triangles) and $l=2$ (squares). The modes with lowest inertia are the ones with higher amplitudes at the surface and hence are easier to observe.

Table 2 Differences in the basic parameters for the models with $1.00 \mathrm{M}_{\odot}$ and $1.20 \mathrm{M}_{\odot}$

\begin{tabular}{ccc}
\hline \hline Mass & $1.00 \mathrm{M}_{\odot}$ & $1.20 \mathrm{M}_{\odot}$ \\
\hline$T_{\text {eff }}$ & $5159 \mathrm{~K}$ & $5190 \mathrm{~K}$ \\
$\log g$ & $2.42 \mathrm{dex}$ & $2.44 \mathrm{dex}$ \\
$R / R_{\odot}$ & 10.2 & 10.9 \\
Age & $5.7 \mathrm{Gyr}$ & $3.2 \mathrm{Gyr}$ \\
\hline \hline
\end{tabular}

In the range of masses investigated by now, the discrepancies in the determination of the basic parameters are showed in Table 2

We also tried to investigate the asymptotic period spacing of the models to see if it would be a good way to disentangle between the mass. However the difference between 1.00 and $1.20 \mathrm{M}_{\odot}$ are of a few seconds, both about $60 \mathrm{~s}$. Bedding et al. (2011) found a value of observed period spacing of $80 \mathrm{~s}$ giving an even larger asymptotic value. The discrepancies in period spacing might be solved with higher mass and is being investigated.

\section{Conclusions and Perspectives}

KIC 7693833 is the so far most metal-poor red giant and it was observed by Kepler during Q1-Q10. From its frequency spectrum, 19 modes of oscillation were identified as $l=$ $0,1,2$ with a $\nu_{\max }=32.2 \mu \mathrm{Hz}$ and $\Delta \nu=4.06 \mu \mathrm{Hz}$.

Models within 1.00-1.20 $\mathrm{M}_{\odot}$ were computed to match the observational constraints using the scaling relations as starting point. The frequencies computed for all models agree within $1.0 \mu \mathrm{Hz}$, having no preferred model. This gives an uncertainty of about $30 \mathrm{~K}$ in $T_{\text {eff }}, 0.02$ dex in $\log g, 0.7$ $R_{\odot}$ in radius, corresponding to $7 \%$, and of about $2.5 \mathrm{Gyr}$ in age. The period spacing of the models computed are smaller than the observed one and the possibility to resolve the discrepancies by possible higher masses is currently investi- 

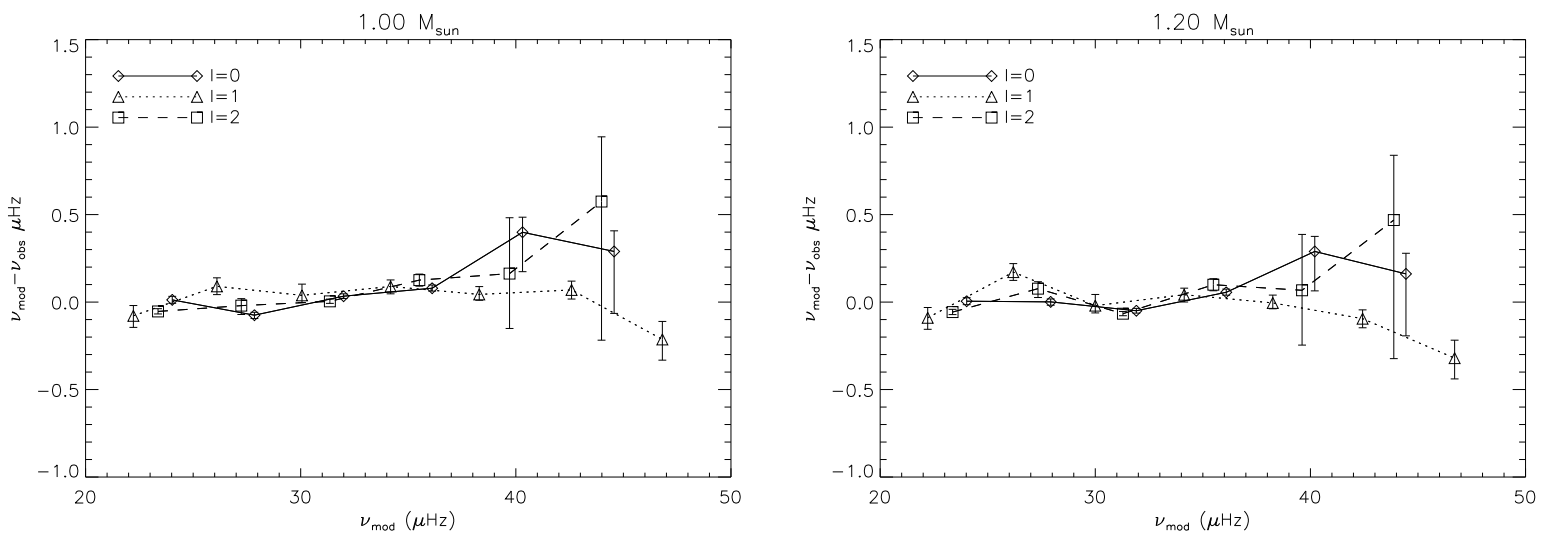

Fig. 2 Frequency difference between model and observational values for models with 1.00 (left) and 1.20 (right) $\mathrm{M}_{\odot}$. Both models agree within a range of $1.0 \mu \mathrm{Hz}$ with the data. There is no preferred model.
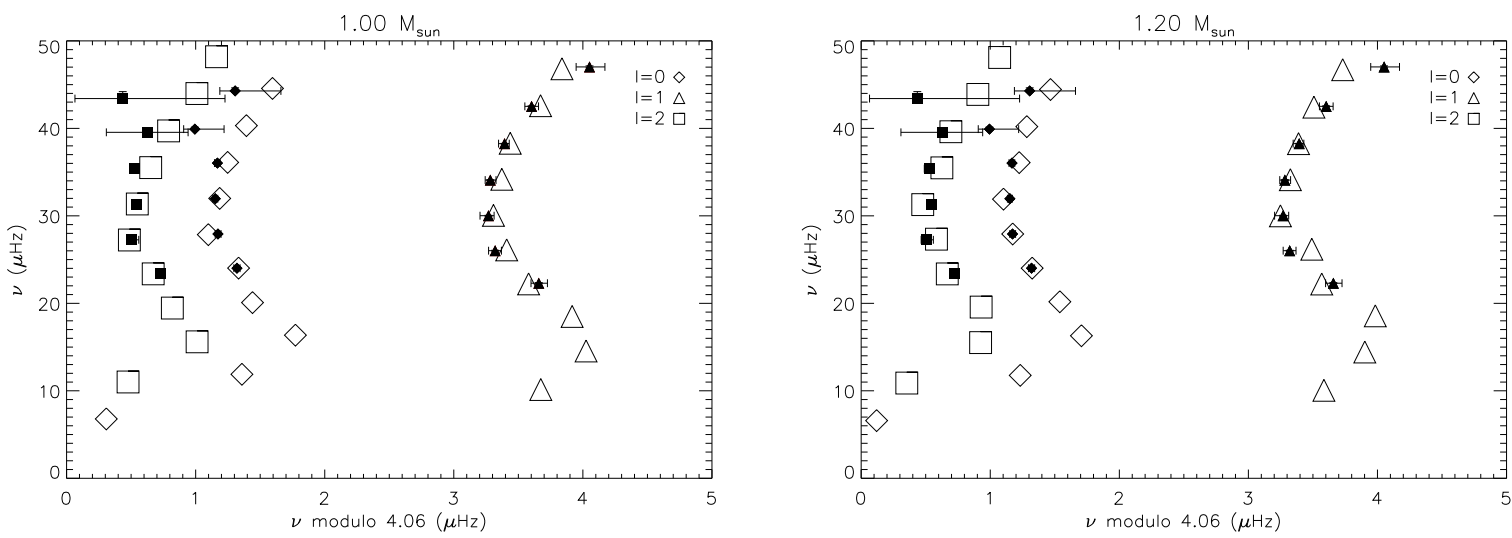

Fig.3 Échelle diagram for the models with $1.00 \mathrm{M}_{\odot}$ (left) and $1.20 \mathrm{M}_{\odot}$ (right). Open symbols show the frequencies computed for the models and filled symbols show the observed ones. Diamonds are for $l=0$ modes, triangles for $l=1$ modes and squares for $l=2$.

gated. This would make the star even younger and quite peculiar for a metal-poor star.

For a more detailed modeling, it is important to investigate different physics inputs and see how the oscillation frequencies change. This way, we might find a way to distinguish between models of different masses and different physics. Currently, we still have no good diagnosis to disentangle models of red giants.

Acknowledgements. Funding for the Kepler Discovery mission is provided by NASA's Science Mission Directorate. Funding for the Stellar Astrophysics Centre is provided by The Danish National Research Foundation. The research is supported by the ASTERISK project (ASTERoseismic Investigations with SONG and Kepler) funded by the European Research Council (Grant agreement no.: 267864).

\section{References}

Aerts C., Christensen-Dalsgaard J., Cunha M., Kurtz D. W., 2008, Sol. Phys., 251, 3

Angulo C., Arnould M., Rayet M., Descouvemont P., et al., 1999, Nuclear Physics A, 656, 3
Bedding T. R., Mosser B., Huber D., et al.., 2011, Nature, 471, 608 Böhm-Vitense E., 1958, ZAp, 46, 108

Brown T. M., Gilliland R. L., Noyes R. W., Ramsey L. W., 1991, ApJ, 368, 599

Christensen-Dalsgaard J., 2008, Ap\&SS, 316, 113

Christensen-Dalsgaard J., Bedding T. R., Kjeldsen H., 1995, ApJ, 443, L29

De Ridder J., Barban C., Baudin F., Carrier F., Hatzes A. P., Hekker S., Kallinger T., Weiss W. W., Baglin A., Auvergne M., Samadi R., Barge P., Deleuil M., 2009, Nature, 459, 398

Di Mauro M. P., Cardini D., Catanzaro G., et al., 2011, MNRAS, 415, 3783

Dupret M.-A., Belkacem K., Samadi R., et al., 2009, A\&A, 506, 57

Kjeldsen H., Bedding T. R., 1995, A\&A, 293, 87

Montalbán J., Miglio A., Noels A., et al., 2010, ApJ, 721, L182

Mosser B., Belkacem K., Goupil M. J., et al., 2011, A\&A, 525, L9

Rogers F. J., Nayfonov A., 2002, ApJ, 576, 1064

Steigman G., 2010, ArXiv e-prints

Thygesen A. O., Frandsen S., Bruntt H., et al., 2012, A\&A, 543, A160

Weiss A., Schlattl H., 2008, Ap\&SS, 316, 99 